\title{
SUVmax of FDG-PET correlates with the effects of neoadjuvant chemoradiotherapy for oral squamous cell carcinoma
}

\author{
AKIHIKO MIYAWAKI ${ }^{1}$, RYUJI IKEDA ${ }^{2}$, HIROSHI HIJIOKA ${ }^{1}$, TAKAYUKI ISHIDA ${ }^{1}$, \\ MINA USHIYAMA ${ }^{2}$, ETSURO NOZOE $^{1}$ and NORIFUMI NAKAMURA ${ }^{1}$ \\ ${ }^{1}$ Department of Oral and Maxillofacial Surgery, Field of Oral and Maxillofacial Rehabilitation, \\ Advanced Therapeutics Course, Graduate School of Medical and Dental Sciences, Kagoshima University; \\ ${ }^{2}$ Department of Clinical Pharmacy and Pharmacology, Kagoshima University Hospital, Kagoshima 890-8520, Japan
}

Received October 19, 2009; Accepted January 20, 2010

DOI: $10.3892 /$ or_00000751

\begin{abstract}
The aim of this study was to analyze the relationship between the maximum standardized uptake value (SUVmax) of 18F-fluoro-2-deoxyglucose-positron emission tomography (FDG-PET) and the effects of neoadjuvant chemoradiotherapy in oral squamous cell carcinoma (OSCC), and to identify the possible biological background of this association. Thirty-seven patients with OSCC, who underwent preoperative FDG-PET followed by cancer treatment with neoadjuvant chemoradiotherapy, were enrolled in this study. The various histological effects following neoadjuvant chemoradiotherapy were compared to the SUVmax in the primary OSCC. These effects were also compared to the immunohistochemical staining score of hypoxia-inducible factor- $1 \alpha$ (HIF- $1 \alpha$ ), glucose membrane transporter (GLUT)-1 and vascular endothelial growth factor (VEGF) in the biopsy specimen. Furthermore, we analyzed the chemosensitivity of KB-3-1 cells to cisplatin under hypoxic conditions using the MTT assay. A negative correlation was observed between the SUVmax and the histological effects following neoadjuvant chemoradiotherapy $(\mathrm{p}<0.01)$. The SUVmax was also correlated with the staining score of HIF- $1 \alpha(\mathrm{p}<0.03)$, but not with GLUT-1 and VEGF. The mean staining score of HIF-1 $\alpha$ in the highly effective group was $2.7 \pm 1.1$, which was significantly lower than that $(3.7 \pm 0.9)$ of the poorly effective group $(\mathrm{p}<0.05)$. The cell chemosensitivity assay revealed chemoresistant effects under a hypoxic condition in OSCC. In conclusion, the SUVmax is correlated with the effectiveness of neoadjuvant chemoradiotherapy in OSCC.
\end{abstract}

Correspondence to: Dr Akihiko Miyawaki, Department of Oral and Maxillofacial Surgery, Graduate School of Medical and Dental Sciences, Kagoshima University, 8-35-1 Sakuragaoka, Kagoshima 890-8520, Japan

E-mail: makihiko@denta.hal.kagoshima-u.ac.jp

Key words: oral squamous cell carcinoma, 18F-fluoro-2-deoxyglucose-positron emission tomography, standardized uptake value, hypoxia-inducible factor- $1 \alpha$, glucose membrane transporter-1, vascular endothelial growth factor
Our clinical and experimental analyses further suggest a possible association of the upregulation of HIF-1 $\alpha$ with chemoradiosensitivity in SCC cells.

\section{Introduction}

The preservation of the morphological characteristics of the oral cavity and its functions such as mastication, swallowing, speech and esthetics is crucially important in the treatment of advanced oral squamous cell carcinoma (OSCC). In the past half century, comprehensive management including neoadjuvant chemotherapy, radiotherapy and surgery has been adopted as the primary treatment of OSCC. Although the tumor-node-metastasis staging system (TNM classification) provides a reliable basis for the determination of patient prognosis and therapeutic planning in OSCC, the clinical or pathological TNM classification does not always provide a satisfactory explanation for differences in relapse and survival, even for patients with the same TNM classification. Squamous cell carcinomas of the head and neck region are histologically distinct but clinically heterogeneous and include multiple anatomical regions with a different history and clinical behavior. The recent accumulation of biological characteristics of head and neck carcinomas based on biological parameters such as tumor aggressiveness and proliferative activity may aid in determining patient prognosis $(1,2)$; however, it is still difficult to precisely predict the effectiveness of chemoradiotherapy and the prognosis of patients using conventional clinicopathological criteria. Therefore, new clinicopathological parameters to predict the effects of neoadjuvant chemoradiotherapy are required.

Due to the difference in the uptake of FDG between benign tumors and malignancies, 18F-fluoro-2-deoxyglucose-positron emission tomography (FDG-PET) is now used extensively as a screening test for cancer, determination of benign tumor or malignancies, staging, the presence of metastasis and detection of residual cancer following treatment $(3,4)$. Although the high FDG uptake in malignant tumors is due to an increased glucose metabolism, the exact mechanism by which FDG accumulates in malignant tumors is not fully understood. However, it has been repeatedly demonstrated that a high standardized uptake value (SUV) is related to an 
inferior overall survival (5-7). Kitagawa et al demonstrated that FDG-PET had a clinical advantage in patient management by facilitating the differentiation of residual tumors from treatment-related changes after chemoradiotherapy in head and neck carcinoma (4). Clinically, FDG-PET has been reported to be useful for evaluation of the therapeutic effects of neoadjuvant chemotherapy and relapse of head and neck cancer (3-6,8-10). In addition, the maximum standardized uptake value (SUVmax) of FDG in the primary tumor has been recognized as an independent prognostic factor for survival in lung cancers, esophageal carcinomas, nasopharyngeal carcinomas and head and neck carcinomas $(5-7,11)$. As mentioned above, a high SUV is correlated with an inferior overall patient survival (5-7).

It has been noted that the tumor microenvironment of many malignancies contains a hypoxic condition, a condition associated with resistance to anticancer agents and poor prognosis (12-14). Hypoxia-inducible factor- $1 \alpha$ (HIF-1 $\alpha$ ) was found to be upregulated in a broad range of tumors and is involved in angiogenesis, invasion and altered glucose metabolism $(14,15)$. Therefore, we focused on the hypoxic condition related to malignant potential and glucose metabolism in malignant tumors. The biological basis of FDG-PET is the greater increase in glucose metabolism in malignant tumors compared with that in normal tissues (16). A variety of mechanisms that might explain the accelerated glucose use in malignant tumors include passive infusion, $\mathrm{Na}^{+}$-dependent glucose transport and via the glucose transporter (GLUT) (17). Furthermore, the tumor microenvironment such as hypoxia might play an important role in the uptake of FDG (7). HIF-1 $\alpha$ is one of the hypoxia-related markers which upregulate the expression of glucose transporters, vascular endothelial growth factor (VEGF) and other target genes. Hypoxia leads to an increase in the rate of glycolysis, which in turn, increases the uptake of FDG.

The aim of the present study was to analyze the correlation between SUVmax in FDG-PET and the effects of neoadjuvant chemoradiotherapy, and to further identify the possible biological background in OSCC. Thus, we investigated the correlation between the SUVmax in FDGPET and the histological effect following neoadjuvant chemoradiation therapy, and the expression of GLUT-1, HIF- $1 \alpha$ as the upstream gene of GLUT-1, and VEGF which are related to tumor angiogenesis.

\section{Materials and methods}

Materials and patient eligibility criteria. Thirty-seven patients, who had been treated in our department between January 2003 and February 2007, were enrolled in this study. There were 28 males and 9 females with a median age of 67.8 years (range $37-83$ years). At least more than one week after incisional biopsy, the patients underwent whole body FDG-PET as part of their routine preoperative staging procedure at the Atsuchi Memorial Clinic PET Center in Japan. The biopsy specimens evaluated were taken from 37 patients with histologically confirmed oral squamous cell carcinoma. We obtained a sufficient amount of biopsy tissue for diagnosis from the lateral portion of the lump including not only tumor tissue but also normal tissue. The volume of the
Table I. Clinical characteristics of the patients.

\begin{tabular}{lrr}
\hline Characteristics & No. of patients $(\%)$ \\
\hline No. of patients & \multicolumn{2}{c}{37} \\
Gender & & \\
Male & 28 & $(75.7)$ \\
Female & 9 & $(24.3)$ \\
Median age in years (range) & 67.8 & $(37-83)$ \\
Primary tumor site & & \\
Tongue & 9 & $(24.3)$ \\
Gingiva & 17 & $(45.9)$ \\
Oral floor & 7 & $(18.9)$ \\
Other regions & 4 & $(10.8)$ \\
Tumor classification & & \\
T1 & 3 & $(8.1)$ \\
T2 & 20 & $(54.1)$ \\
T3 & 7 & $(18.9)$ \\
T4a & 7 & $(18.9)$ \\
Stage & & \\
I & 2 & $(5.4)$ \\
II & 10 & $(27.0)$ \\
III & 10 & $(27.0)$ \\
IVA & 15 & $(40.5)$ \\
\hline
\end{tabular}

Thirty-seven patients, who had been treated in our department between January 2003 and February 2007, were enrolled in this study. All patients underwent FDG-PET/CT before treatment at the Atsuchi Memorial Clinic PET Center in Japan. No patients presented with diabetes mellitus.

fusiform-shaped biopsy tissues was $\sim 0.5 \mathrm{~cm}^{3}$. Written informed consent was obtained from all patients.

The clinical staging was based on the International Union Against Cancer TNM classification (2002, 6th edition). Computed tomography and magnetic resonance imaging studies were performed on all patients before treatment. Twenty six patients out of 37 who were diagnosed with greater or equal to early T2 (3 cm or more) basically received neoadjuvant chemoradiotherapy as an organ preservation. This included external beam radiation therapy with irradiation of 30 Gy average in 15 fractions and concurrent chemotherapy of either platinum-containing drugs such as cisplatin $(60 \mathrm{mg}$ / $\mathrm{m}^{2}$ ) or carboplatin (AUC 4) on day 1 and 5-fluorouracil $\left(500 \mathrm{mg} / \mathrm{m}^{2}\right.$, day 1-5) intravenously. Approximately 2 weeks after the chemoradiotherapy, the patients underwent definitive surgery. The clinical characteristics of the study population are summarized in Table I.

18F-FDG PET. FDG-PET images were obtained on a Discovery ST product by the General Electric Corporation. The patients were instructed to refrain from ingesting processed sugar for $6 \mathrm{~h}$ before PET scanning, and the exclusion criteria included diabetes mellitus. None of the patients were extremely obese. 
Table II. Histological grading of the effect of chemoradiotherapy.

Grade

Histological findings

I Characteristic changes are noted in tumor cells, but tumor structures have not been destroyed. (There is no detection of tumor nests resulting from lysis in individual tumor cells).

II In addition to characteristic cellular changes, tumor structures have been destroyed as a result of the disappearance of tumor cells. However, a variable number of 'viable cells' remain.

IIa Destruction of tumor structures is of a mild degree (i.e. 'viable tumor cells' are frequently observed). IIb Destruction of tumor structures is of a severe degree (i.e. 'viable tumor cells' are few in number).

III Markedly altered, presumably no viable tumor cells are present singly or in small clusters, and 'viable tumor cells' are rarely observed.

IVA No tumor cells remain in any sections (local cure).

We applied the classification reported by Shimosato et al (18) to the histologic effect of preoperative chemoradiation therapy. Patients who exhibited a histological effect following neoadjuvant chemoradiotherapy more effective than or equal to grade IIb was defined as the highly effective group, and patients with an effect lower than or equal to grade IIa were included in the poorly effective group.

Whole body scanning was performed $1 \mathrm{~h}$ after FDG dosing, and subsequently, the local image was scanned $2 \mathrm{~h}$ later. The FDG dose was $3.7 \mathrm{MBq} / \mathrm{kg}$ by weight. After 2-D whole body emission images were acquired, images were reconstructed using an interactive reconstruction algorithm. For determination of SUV, a region of interest (ROI) was drawn by the radiologists on the transaxial images around the primary tumor on demonstration of an enhanced CT. SUV was then automatically calculated as the activity concentration of FDG uptake divided by the FDG injected dose/body weight (g). The SUVmax equals the organic radioactivity $(\mathrm{MBq} / \mathrm{g}) / \mathrm{FDG}$ dose $(\mathrm{MBq})$ /body weight $(\mathrm{g})$.

Correlation between the SUVmax and the histological effect following neoadjuvant chemoradiotherapy. The histological effects of chemoradiotherapy using H\&E-stained excised specimens of 26 patients who received neoadjuvant chemoradiotherapy were evaluated retrospectively. Assessment of the histological effects complied with the classification reported by Shimosato et al (18) (Table II).

Correlation between the SUVmax and immunohistochemical staining score and assessment of the immunohistochemical staining score. The correlation between the SUVmax in the primary OSCC and the immunohistochemical staining score of HIF-1 $\alpha$, GLUT-1 and VEGF was evaluated using biopsy specimens of OSCC. All OSCC biopsy specimens were fixed routinely in $10 \%$ formalin, embedded in paraffin and sectioned into $4-\mu \mathrm{m}$ slices. The immunostaining procedure was performed with Envision Kit/HRP (Dako Glostrup, Denmark). After deparaffinization and hydration, the endogenous peroxidase activity was blocked by immersing the specimens in a $0.3 \%$ hydrogen peroxide-methanol solution for $30 \mathrm{~min}$ at room temperature. In preparation for staining with anti-HIF-1 $\alpha$ and anti-VEGF antibodies, specimens were treated with $0.01 \mathrm{M}$ citrate buffer $(\mathrm{pH} 6.0)$ for $10 \mathrm{~min}$ at $121^{\circ} \mathrm{C}$ in an autoclave, and GLUT-1 was treated with $0.01 \mathrm{M}$ citrate buffer ( $\mathrm{pH}$ 6.0) for $5 \mathrm{~min}$ in a microwave oven (strong range). The sections were incubated with either mouse monoclonal anti-
Table III. Immunohistochemical staining score

\begin{tabular}{lccc}
\hline Intensity & Score & Positive cells & Score \\
\hline Negative & 0 & $0 \%$ & 0 \\
Weak & 1 & $<25 \%$ & 1 \\
Intermediate & 2 & $\leq 50 \%$ & 2 \\
Strong & 3 & $>50 \%$ & 3
\end{tabular}

To evaluate the immunohistochemical expression, a score corresponding to the sum of both a) intensity and b) percentage of positive cells, as described elsewhere, was established following Shibusa et al (19). A score $>3$ represented a positive immunohistochemical result.

human HIF-1 $\alpha$ antibody (1:200 dilution) (BD Bioscience), mouse monoclonal anti-human GLUT-1 antibody (1:500 dilution) (R\&D Systems) and rabbit polyclonal anti-human VEGF antibody (1:100 dilution) (Santa Cruz) diluted in PBS at $4{ }^{\circ} \mathrm{C}$ overnight. After incubation with the primary antibodies, the section was incubated with the appropriate secondary antibodies conjugated with peroxidase-labeled dextran polymers for $40 \mathrm{~min}$. After rinsing in PBS, the immune complex was visualized by incubating the sections with diaminobenzene tetrahydrochloride. The sections were rinsed briefly in water and counterstained with Mayer's hematoxylin. Negative controls for the immunostaining procedure were prepared by omission of the primary antibody.

Positive expression of HIF-1 $\alpha$, GLUT-1 and VEGF was determined as follows. Tumor sections were observed under high-power (x400) fields. The number of fields observed was dependent on the amount of viable tissue available, and fields were matched for each antibody marker. The number of tumor cells stained divided by the total number of tumor cells was expressed as a percentage. To evaluate the immunohistochemical expression, a score corresponding to the sum of both a) intensity and b) percentage of positive cells, as described elsewhere, was established following Shibusa et al (19) 


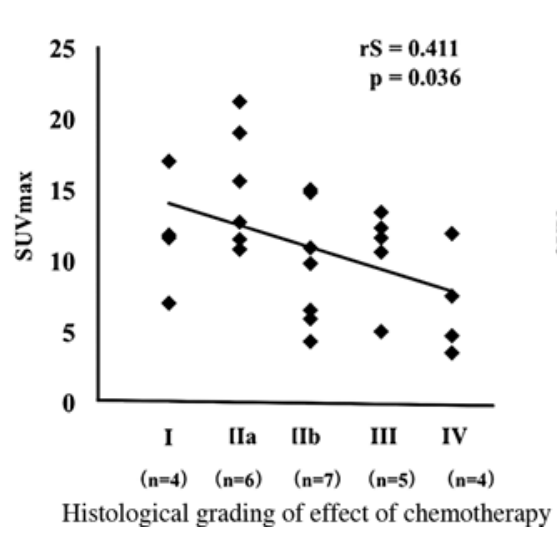

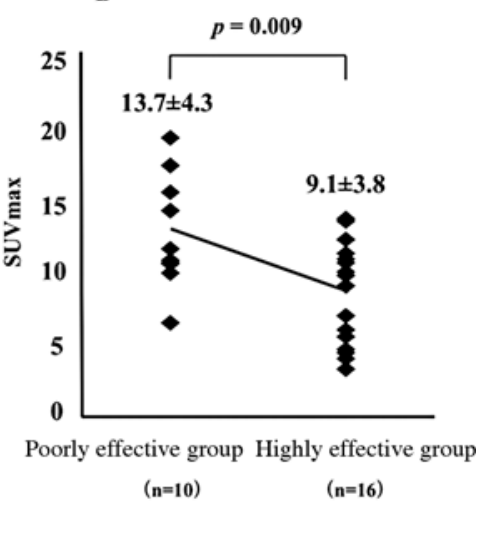

Figure 1. Correlation between the SUVmax and the histologic effect of neoadjuvant chemoradiation therapy. A higher SUVmax in the primary OSCC was significantly correlated with poor histological effects following neoadjuvant chemoradiotherapy.
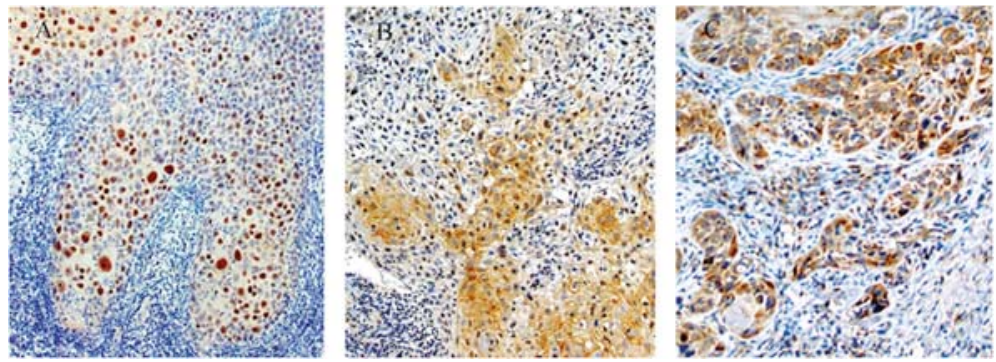

Figure 2. Expression of HIF-1 $\alpha$, GLUT-1 and VEGF in OSCC. Positive expression of HIF-1 $\alpha$ was noted in the nuclei of OSCC (A). Positive expression of GLUT-1 was noted in the cytosol and membrane of OSCC (B). Positive expression of VEGF was noted in the cytosol of OSCC (C).

(Table III). A score $>3$ represented a positive immunohistochemical result.

Correlation between the histological effect following neoadjuvant chemoradiotherapy and the immunohistochemical staining score. The correlation between the histological effects following neoadjuvant chemoradiotherapy and the immunohistochemical staining scores of HIF-1 $\alpha$, GLUT-1 and VEGF was investigated using biopsy specimens of OSCC.

Inhibitory effect of cisplatin under hypoxia as determined using the MTT assay. The MTT colorimetric assay was used to assess the sensitivity of cells to agents in vitro as described elsewhere (20). Exponentially growing cells were trypsinized and harvested, and equal numbers of cells in $180 \mu 1$ of MEM were inoculated into each well of a 96-well microplate. After incubating overnight, $20 \mu \mathrm{l}$ of cisplatin was added to the culture, and cells were incubated for 3 days under normoxic and hypoxic conditions. Surviving cells were determined as previously described (21). The $\mathrm{IC}_{50}$ was measured as the concentration of agents that reduced the number of cells to $50 \%$ of that in the control medium.

Statistical analysis. Spearman's rank correlations were used to estimate associations between the different continuous parameters (SUVmax) and ordinal parameters (histological effect, immunohistochemical score of HIF-1 $\alpha$, GLUT-1 and VEGF). Non-parametric statistics (Mann-Whitney calibration test) were used to test whether FDG uptake was different based on a categorical parameter (poorly effective group versus highly effective group). The Student's t-test was used to compare the difference between the population mean value of the poorly effective group versus the highly effective group. All statistical evaluations were performed with Excel statistical software. p-values $<0.05$ were considered statistically significant.

\section{Results}

Correlation between the SUVmax and the histological effect following neoadjuvant chemoradiotherapy. The relationship between the SUVmax and the histological effect of neoadjuvant chemoradiotherapy is shown in Fig. 1. The SUVmax was significantly lower with higher histological grade following neoadjuvant chemoradiotherapy (Fig. 1A). Patients who had a histological effect following neoadjuvant chemoradiotherapy more effective than or equal to grade IIb were classified as the highly effective group, and those with a histological effect lower than or equal to grade IIa were included in the poorly effective group. The mean SUV in the highly effective group was $9.1 \pm 3.8$ while that in the poorly effective group was $13.7 \pm 4.3$, which was significantly different $(\mathrm{p}<0.01)$ as shown in Fig. 1B. A higher SUVmax in 

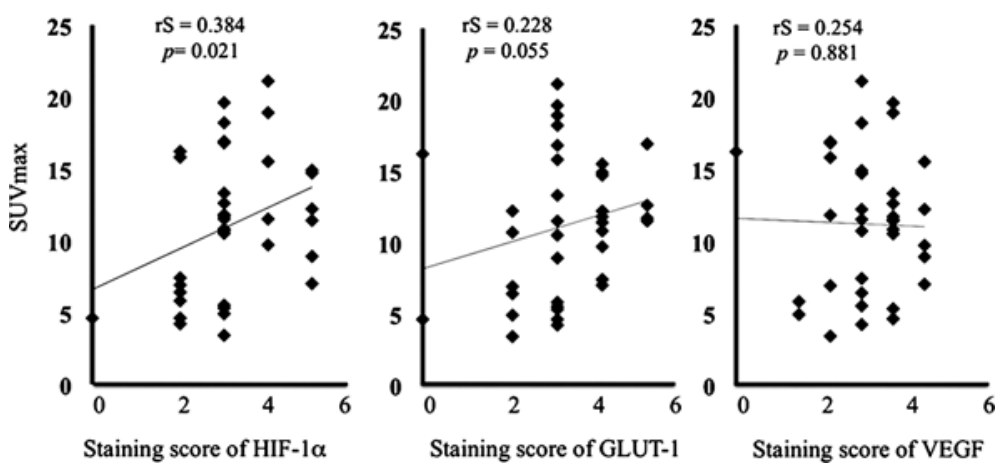

Figure 3. Correlation between the SUVmax and the staining score of HIF-1 $\alpha$, GLUT-1 and VEGF. The SUVmax was correlated with the staining score of HIF-1 $\alpha$ but not with the expression of GLUT-1 and VEGF.

$\left.\begin{array}{l}\text { high effect group: average score } 2.7 \\ \text { low effect group: average score } 3.7\end{array}\right] p=0.05$

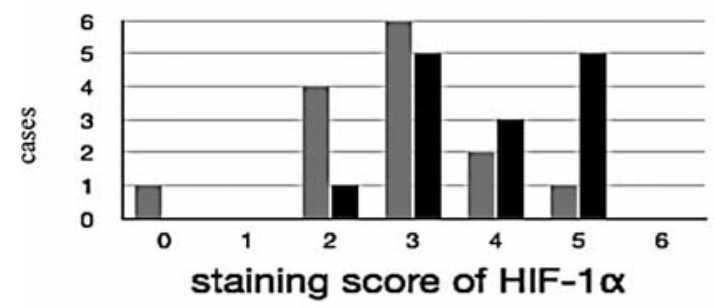

high effect group: average score $3.17 \mathrm{~ns}$ low effect group: average score 3.4

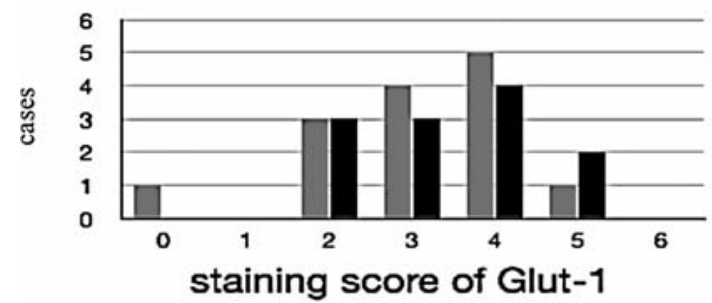

high effect group: average score $4.1-\mathrm{ns}$ low effect group: average score 4.6

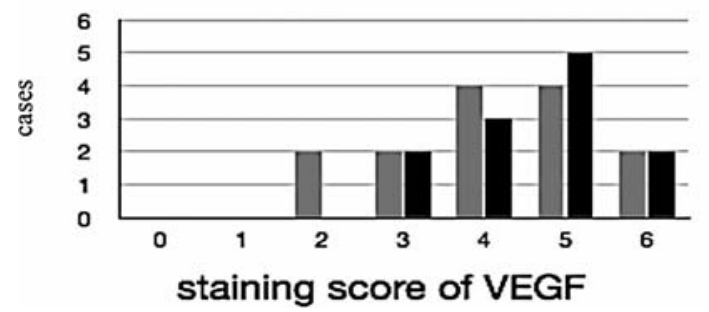

Figure 4. Correlation between the histological effects of neoadjuvant chemoradiotherapy and the immunostaining score of HIF-1 $\alpha$, GLUT-1 and VEGF. A higher staining score of HIF-1 $\alpha$ was correlated with a poor histological effect following neoadjuvant chemoradiotherapy $(\mathrm{p}<0.01)$. However, the staining score of GLUT-1 and VEGF was not associated with histological effect.

the primary OSCC was significantly associated with a poor histological effect following neoadjuvant chemoradiotherapy.
Correlation between the SUVmax and the immunohistochemical staining score. Immunohistologically, clusters of HIF-1 $\alpha$-positive cells were observed within the individual tumors, including the nuclei of the tumor cells (Fig. 2A). Immunohistochemical expression of HIF-1 $\alpha$ was found in 29 of the 37 cases $(78.3 \%)$. Expression of GLUT-1 was noted within the individual tumors, including the membrane and cytosol of the tumor cells (Fig. 2B), and was noted in 30 of the 37 cases $(80.0 \%)$. Expression of VEGF was also observed within the individual tumors, including the cytosol of the tumor cells (Fig. 2C), and was observed in 35 of the 38 cases (94.5\%). Clusters of VEGF-positive cells were most dense at the tumor margins (Fig. 2C). As shown in Fig. 3, the staining score of HIF- $1 \alpha$ was significantly $(p<0.03)$ positively associated with the SUVmax. In addition, the staining score of GLUT-1 tended to be associated with the SUVmax $(\mathrm{p}=0.055)$; however, the staining score of VEGF was not correlated.

Correlation between the histological effect and the immunohistochemical staining score. Distribution of the cases to the highly effective group or the poorly effective group according to the staining score of HIF-1 $\alpha$, GLUT- 1 and VEGF is shown in Fig. 4. The results showed that a higher staining score of HIF-1 $\alpha$ was related to a poor histological effect following neoadjuvant chemoradiotherapy $(\mathrm{p}<0.05)$; however, expression of GLUT-1 and VEGF was not related to the histological effect.

Inhibitory effect of hypoxia on the cytotoxicity of cisplatin. Hypoxic cancer cells undergo a series of genetic and metabolic alterations that allow them to survive and become more resistant to chemotherapy and radiotherapy via the HIF- $1 \alpha$ pathway $(12,13)$. We, therefore, investigated the effects of hypoxia on the drug sensitivity of KB-3-1 cells (a human epidermoid $\mathrm{KB}$ carcinoma cell line) by comparing the sensitivity of KB-3-1 cells in normoxic and hypoxic environments. Sensitivity to cisplatin was determined using the MTT assay. $\mathrm{IC}_{50}$ values for cisplatin were 4.0 and $15.7 \mu \mathrm{M}$ for KB-3-1 cells under normoxic and hypoxic conditions, respectively. Under hypoxic conditions, KB-3-1 cells were $\sim 4$ times more resistant to cisplatin than under normoxic conditions (Fig. 5). This finding suggests that hypoxia increases resistance to cisplatin in KB-3-1 cells, and that an 


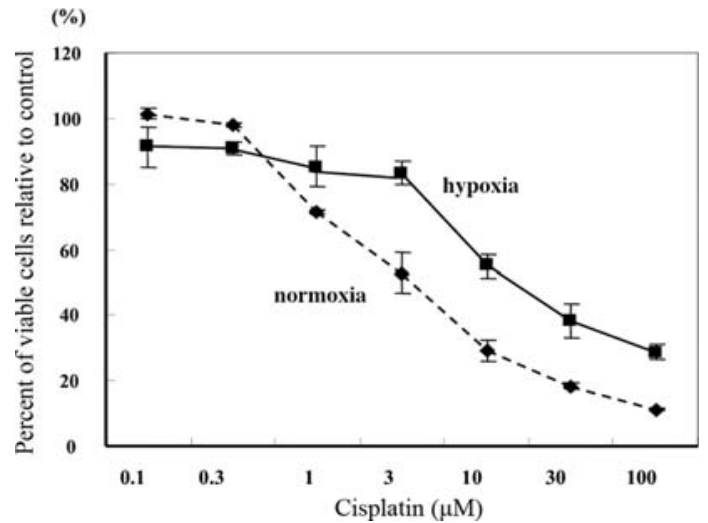

Figure 5. Chemosensitivity assay (MTT assay). Hypoxia led to an increase in the survival of KB-3-1 cells after exposure to cisplatin. KB-3-1 cells were $\sim 4$ times more resistant to cisplatin than under normoxic condition.

ineffective response to preoperative chemoradiotherapy for OSCC might be related to tumor hypoxia.

\section{Discussion}

FDG uptake of FDG-PET is based on the increased glucose use in malignant tumors compared to that in normal tissues. Recent research indicates that the tumor microenvironment such as hypoxia might play an important role in the uptake of FDG (7) involving a variety of mechanisms. Hypoxia is also associated with resistance to chemotherapeutic drugs and poor prognosis (12-14). Thus, the present study investigated the correlations between the SUVmax of FDG-PET, the histological effect following neoadjuvant chemoradiotherapy, and expression of the hypoxia-related marker HIF-1 $\alpha$ which upregulates GLUT-1 and VEGF in OSCC. In addition, this study aimed to estimate the role of the SUVmax as a new clinicopathological indicator to predict the effects of neoadjuvant chemoradiotherapy.

Several conclusions were derived from our clinical and experimental analyses. The SUVmax was associated with the histological effects of neoadjuvant chemoradiotherapy and hypoxia was correlated with a higher uptake of FDG in OSCC. Additionally, the expression of HIF-1 $\alpha$ related to a hypoxic condition was correlated with poor chemoradiosensitivity. These correlations explained why the SUVmax was associated with the histological effects of neoadjuvant chemoradiotherapy in OSCC.

First, a positive correlation was noted between the SUVmax in the primary OSCC and the histological effect of neoadjuvant chemoradiotherapy, but there was an overlap between the SUVmax in the highly effective group and that in the poorly effective group (Fig. 1B). For this reason, several factors such as tumor size, inflamed tumor and the site of tumor were considered (22). Therefore, when the clinical characteristics of patients were similar, the overlap of SUVmax was minimized. However, a high SUV has been reported as a poor prognostic factor in other malignancies including esophageal, nasopharyngeal, lung and head and neck carcinomas $(5,10,11,23,24)$. In addition, it has been reported that a higher FDG uptake can partially predict the

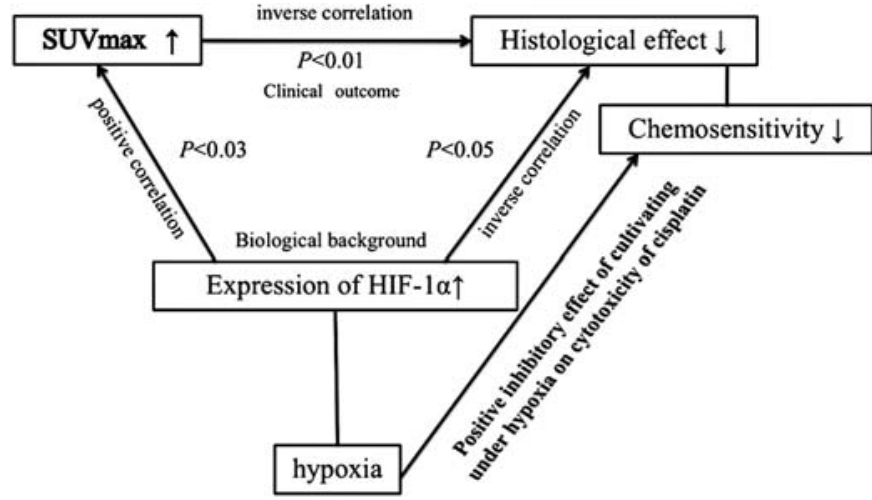

Figure 6. Correlation among the histological effect of chemoradiotherapy, SUVmax and expression of HIF-1 $\alpha$. The SUVmax of FDG-PET in the primary OSCC was correlated with the histological effects and with the staining score of HIF-1 $\alpha$. Furthermore, the histological effect was correlated with the staining score of HIF-1 $\alpha$. Based on our experimental analyses, hypoxia led to chemoresistance in SCC cells. These findings suggest a possible association of HIF-1 $\alpha$ upregulation with their correlation as a biological mechanism.

response to concomitant chemotherapy in OSCC (3), although there have been few reports concerning the prediction of the histological response to neoadjuvant chemoradiotherapy. These reports support our main conclusion that a high SUVmax is associated with a poor effect following neoadjuvant chemoradiotherapy (Fig. 1A and B). Although prognostic factors for cancer treatment have been identified by the immunohistochemical staining of tumor tissues or tissue microarrays as well as morphological examination, biopsy specimens may not represent the morphological alteration or genetic information of the entire malignant tumor $(22,25)$. However, FDG-PET imaging may provide information regarding the metabolic change in the entire tumor. Therefore, the SUVmax might serve as an indicator of the histological effects following chemoradiotherapy, and may lead to individualized treatment in OSCC.

Secondly, in regard to the correlation between uptake of FDG and the immunostaining score, activation of the glycolytic metabolism was established as a characteristic of malignancies in a basic study by Warburg (16). Increasing glycolytic metabolism has been shown to depend above all on the glycolytic phenotype, overexpression of glucose transporters, particularly of GLUT-1, in a variety of tumor types, including OSCC (26). In addition, overexpression of GLUT-1 is detected at an early stage after cancer transformation (27). Furthermore, a promotive effect through the HIF- $1 \alpha$-binding site of the GLUT-1 promoter region has been identified. The hypoxic condition in tumors such as non-small cell lung cancer (NSCLS) plays an important role in the uptake of FDG (7), leading to the upregulation of GLUT-1, VEGF and other target genes via the HIF- $1 \alpha$ pathway. As a result, the uptake of FDG increases. These facts corroborate our finding that a high uptake of FDG is correlated with an overexpression of HIF-1 $\alpha$ (Fig. 3). However, the SUVmax tended to be associated with the staining score of GLUT-1 but not with that of VEGF. Although biopsy specimens provide information concerning protein expression in a limited part of the OSCC specimen, 
the reasons might be that the positive rate of immunostaining of GLUT-1 and VEGF was greater than that of HIF-1 $\alpha$, and furthermore the molecular pathways leading to VEGF upregulation are not just limited to HIF.

Third, our results showed that a poor histological effect following neoadjuvant chemoradiotherapy was related to upregulation of HIF-1 $\alpha$, but clinically, the correlation between expression of GLUT-1 and VEGF and a poor histological effect was unclear. For this reason, it was suggested that the predictive factors of the histological effect following neoadjuvant chemoradiotherapy are not determined by immunostaining score alone, and the histological effects are related to the location and size of the tumor and other clinical and morphological factors (1). On the other hand, HIF-1a and GLUT-1 are negative biomarkers of prognosis and risk factors of recurrence in malignant tumors including OSCC (26) and NSCLS (24). Hypoxic cancer cells undergo a series of genetic and metabolic alterations that allow them, not only to survive, but also to become more resistant to chemotherapy and radiotherapy via the HIF-1 $\alpha$ pathway $(12,13)$.

Furthermore, we investigated the effects of a hypoxic condition on the drug sensitivity of KB-3-1 cells (head and neck squamous cell carcinoma). The sensitivity of KB-3-1 to cisplatin was compared under normoxic or hypoxic conditions. Sensitivity to cisplatin was determined using the MTT assay. The $\mathrm{IC}_{50}$ values for cisplatin were 4.0 and $15.7 \mu \mathrm{M}$ for KB-3-1 cells under normoxic and hypoxic conditions, respectively. Under a hypoxic condition, KB-3-1 cells were approximately 4 times more resistant to cisplatin than under a normoxic condition (Fig. 5). This finding suggests that hypoxia leads to cisplatin resistance in KB-3-1 cells, and a poor histological effect following neoadjuvant chemoradiation therapy in OSCC is correlated with a tumor hypoxic condition.

Based on the above findings, the amount of FDG uptake as measured by SUVmax is correlated with the histological effects of neoadjuvant chemoradiotherapy. An illustrated summary of our clinical and experimental analyses is shown in Fig. 6. The possible biological mechanism involved may be upregulation of HIF- $1 \alpha$ and GLUT-1. The relationship between histological effect and expression of HIF-1 $\alpha$ was also supported by a decrease in chemosensitivity of cultured KB-3-1 cells under hypoxia. Therefore, we found that FDGPET provides biological characterization of OSCC and might be beneficial in cancer treatment. Additionally, a hypoxic tumor microenvironment is possibly related to the SUVmax in OSCC. Combining the SUVmax with other clinical prognostic factors, morphological and molecular prognostic factors might provide useful information for the treatment of cancer. In the future, when specific diagnostic agents (28-30) for hypoxic conditions on PET-CT can be used in OSCC, FDGPET can be considered a conventional and non-invasive imaging modality for predicting the histological effects following neoadjuvant chemoradiotherapy. However, further study is needed to evaluate the specificity between the SUVmax, hypoxia condition and the histological effect of neoadjuvant chemoradiotherapy in additional cases of OSCC

In conclusion, the SUVmax is correlated with the effectiveness of neoadjuvant chemoradiotherapy in OSCC. Our clinical and experimental analyses further suggest a possible association of the upregulation of HIF-1 $\alpha$ as the biological mechanism.

\section{Acknowledgements}

The authors thank Dr Jinnouchi Masashi of the Atsuchi Memorial Clinic PET Center for assistance with the FDG$\mathrm{PET} / \mathrm{CT}$ imaging and diagnoses.

\section{References}

1. Shah NG, Trivedi TI, Rajen A, et al: Prognostic significance of molecular markers in oral squamous cell carcinoma: multivariable analysis. Head Neck 31: 1544-1556, 2009.

2. Uehara M, Sano K, Ikeda H, et al: Hypoxia-inducible factor 1 alpha in oral squamous cell carcinoma and its relation to prognosis. Oral Oncol 45: 241-246, 2009.

3. Kitagawa Y, Sadato N, Azuma H, et al: FDG PET to evaluate combination intra-arterial chemotherapy and radiotherapy of head and neck neoplasms. J Nucl Med 40: 1132-1137, 1999.

4. Kitagawa Y, Nishizawa S, Sano K, Ogasawara T, et al: Prospective comparison of $18 \mathrm{~F}-\mathrm{FDG}$ PET with conventional imaging modalities (MRI, CT and $67 \mathrm{Ga}$ scintigraphy) in assessment of combined intraarterial chemotherapy and radiotherapy for head and neck carcinoma. J Nucl Med 44: 198-206, 2003.

5. Brun E, Kjellen E, Tennvall J, et al: FDG-PET studies during treatment: Prediction of therapy outcome in head and neck squamous carcinoma. Head Neck 24: 127-135, 2002.

6. Swisher SG, Erasmus J, Maish M, et al: 2-Fluoro-2-deoxy-Dglucose positron emission tomography imaging is predictive of pathologic response and survival after preoperative chemoradiation in patients with esophageal carcinoma. Cancer 101: 1776-1785, 2004

7. Van Baardwijk A, Dooms C, van Suylen RJ, et al: The maximum uptake of $18 \mathrm{~F}$-deoxyglucose on positron emission tomography scan correlates with survival, hypoxia inducible factor- $1 \alpha$ and GLUT-1 in non-small cell lung cancer. Eur J Cancer 43: 1392-1398, 2007.

8. Li SJ, Guo W, Ren GX, Huang G, Chen T and Song SL: Expression of Glut-1 in primary and recurrent head \& neck squamous cell carcinomas, and compared with 2-[18F]fluoro-2deoxy-D-glucose accumulation in positron emission tomography. Br J Oral Maxillofac Surg 46: 180-186, 2008.

9. Lowe VJ, Dunphy FR, Varvares M, et al: Evaluation of chemotherapy response in patients with advanced head and neck cancer using [F-18]fluorodeoxyglucose positron emission tomography. Head Neck 19: 666-674, 1997.

10. Kitagawa Y, Sano K, Nishizawa S, et al: FDG PET for prediction of tumor aggressiveness and response to intra-arterial chemotherapy and radiotherapy in head and neck cancer. Eur J Nucl Med 30: 63-71, 2003.

11. Lee SW, Nam SY, Im KC, et al: Prediction of prognosis using standardized uptake value of 2-[(18)F]fluoro-2-deoxy-D-glucose positron emission tomography for nasopharyngeal carcinomas. Radiother Oncol 87: 211-216, 2008.

12. Harada H, Kizaka-Kondoh S, Li G, et al: Significance of HIF-1active cells in angiogenesis and radioresistance. Oncogene 26: 7508-7516, 2007.

13. Song X, Liu X, Chi W, et al: Hypoxia-induced resistance to cisplatin and doxorubicin in non-small cell lung cancer is inhibited by silencing of HIF- $1 \alpha$ gene. Cancer Chemother Pharmacol 58: 776-784, 2006.

14. Lin PY, Yu CH, Wang JT, et al: Expression of hypoxiainducible- $1 \alpha$ is significantly associated with the progression and prognosis of oral squamous cell carcinomas in Taiwan. J Oral Pathol Med 37: 18-25, 2008.

15. Zhong H, De Marzo AM, Laughner E, et al: Overexpression of hypoxia-inducible- $1 \alpha$ in common human cancer and their metastases. Cancer Res 59: 5830-5835, 1999.

16. Kim JW and Dang CV: Cancer's molecular sweet tooth and the Warburg effect. Cancer Res 66: 8927-8930, 2006.

17. Tian M, Zhang H, Nakasone Y, Mogi K and Endo K: Expression of Glut-1 and Glut-3 in untreated oral squamous cell carcinoma compared with FDG accumulation in a PET study. Eur J Nucl Med Mol Imaging 31: 5-12, 2004. 
18. Shimosato Y, Oboshi S and Baba K: Histological evaluation of effects of radiotherapy and chemotherapy for carcinoma. J Clin Oncol 1: 19-35, 1971.

19. Shibusa T, Shijubo N and Abe S: Tumor angiogenesis and vascular endothelial growth factor expression in stage 1 lung adenocarcinoma. Clin Cancer Res 4: 1483-1487, 1998

20. Carmichael J, DeGraff WG, Gazdar AF, Minna J and Mitchell JB: Evaluation of a tetrazolium-based semiautomated colorimetric assay: assessment of chemosensitivity testing. Cancer Res 47: 936-942, 1987.

21. Chen Z-S, Mutoh M, Sumizawa T, Furukawa T, et al: An active efflux system for heavy metals in cisplatin-resistant human KB carcinoma cells. Exp Cell Res 240: 312-320, 1998.

22. De Geus-Oei LF, van Krieken JH, Aliredjo RP, et al: Biological correlates of FDG uptake in non-small cell lung cancer. Lung Cancer 55: 79-87, 2007.

23. Kim MK, Ryu JS, Kim SB, et al: Value of complete metabolic response by $18 \mathrm{~F}$-fluorodeoxyglucose-positron emission tomography in oesophageal cancer for prediction of pathologic response and survival after preoperative chemoradiotherapy. Eur J Cancer 43: 1385-1392, 2007.

24. Higashi K, Ueda Y, Arisaka Y, et al: 18F-FDG uptake as a biologic prognostic factor for recurrence in patients with surgically resected non-small cell lung cancer. J Nucl Med 43: $39-45,2002$
25. Yamamoto E, Kohama G, Sunakawa H, Iwai M and Hiratsuka H: Mode of invasion, bleomycine sensitivity, and clinical course in squamous cell carcinoma of the oral cavity. Cancer 51: 21752180,1983

26. Kunkel M, Moergel M, Stockinger M, et al: Overexpression of Glut-1 is associated with resistance to radiotherapy and adverse prognosis in squamous cell carcinoma of the oral cavity. Oral Oncol 43: 796-803, 2007.

27. Cantuaria G, Magalhaes A, Penalver M, et al: Expression of GLUT-1 glucose transporter in borderline and malignant epithelial tumor of ovary. Gynecol Oncol 79: 33-37, 2000.

28. Mees G, Dierckx R, Vangestel C and Van de Wiele C: Molecular imaging of hypoxia with radiolabelled agents. Eur J Nucl Med Mol Imaging 36: 1674-1686, 2009.

29. Oriuchi N, Higashi T, Ishikita T, et al: Present role and future prospects of positron emission tomography in clinical oncology. Cancer Sci 97: 1291-1297, 2006.

30. Saga T, Koizumi M, Furukawa T, Yoshikawa K and Fujibayashi Y: Molecular imaging of cancer evaluating characters of individual cancer by PET/SPECT imaging. Cancer Sci 100: 375-381, 2009. 